\title{
Atmiyata, a community-led intervention to address common mental disorders: Study protocol for a stepped wedge cluster randomized controlled trial in rural Gujarat, India
}

Kaustubh Joag ${ }^{1 *}$ (D), Jasmine Kalha ${ }^{1}$, Deepa Pandit ${ }^{1}$, Susmita Chatterjee ${ }^{2}$, Sadhvi Krishnamoorthy ${ }^{1}$, Laura Shields-Zeeman ${ }^{3}$ and Soumitra Pathare ${ }^{1}$

\begin{abstract}
Background: While lay-health worker models for mental health care have proven to be effective in controlled trials, there is limited evidence on the effectiveness and scalability of these models in rural communities in low- and middle-income countries (LMICs). Atmiyata is a rural community-led intervention using local community volunteers, called Champions, to identify and provide a package of community-based interventions for mental health, including evidence-based counseling for persons with common mental disorders (CMD).

Methods: The impact of the Atmiyata intervention is evaluated through a stepped wedge cluster randomized controlled trial (SW-CRCT) with a nested economic evaluation. The trial is implemented across 10 sub-blocks (645 villages) in Mehsana district in the state of Gujarat, with a catchment area of 1.52 million rural adults. There are 56 primary health centers (PHCS) in Mehsana district and villages covered under these PHCs are equally divided into four groups of clusters of 14 PHCs each. The intervention is rolled out in a staggered manner in these groups of villages at an interval of 5 months.

The primary outcome is symptomatic improvement measured through the GHQ-12 at a 3-month follow-up. Secondary outcomes include: quality of life using the EURO-QoL (EQ- 5D), symptom improvement measured by the Self-Reporting Questionnaire-20 (SRQ-20), functioning using the World Health Organization's Disability Assessment Scale (WHO-DAS-12), depression symptoms using the Patient Health Questionnaire (PHQ-9), anxiety symptoms using Generalized Anxiety Disorder Questionnaire (GAD-7), and social participation using the Social Participation Scale (SPS). Generalized linear mixed effects model is employed for binary outcomes and linear mixed effects model for continuous outcomes. A Return on Investment (ROI) analysis of the intervention will be conducted to understand whether the intervention generates any return on financial investments made into the project.

Discussion: Stepped wedge designs are increasingly used a design to evaluate the real-life effectiveness of interventions. To the best of our knowledge, this is the first SW-CRCT in a low- and middle-income country evaluating the impact of the implementation of a community mental health intervention. The results of this study will contribute to the evidence on scaling-up lay health worker models for mental health interventions and contribute to the SW-CRCT literature in low- and middle-income countries.

(Continued on next page)
\end{abstract}

\footnotetext{
* Correspondence: kaustubh@cmhlp.org

${ }^{1}$ Centre for Mental Health Law and Policy, Indian Law Society, Law College

Road, Pune 411004, India

Full list of author information is available at the end of the article
}

(c) The Author(s). 2020 Open Access This article is distributed under the terms of the Creative Commons Attribution 4.0 International License (http://creativecommons.org/licenses/by/4.0/), which permits unrestricted use, distribution, and reproduction in any medium, provided you give appropriate credit to the original author(s) and the source, provide a link to the Creative Commons license, and indicate if changes were made. The Creative Commons Public Domain Dedication waiver (http://creativecommons.org/publicdomain/zero/1.0/) applies to the data made available in this article, unless otherwise stated. 
(Continued from previous page)

Trial registration: The trial is registered prospectively with the Clinical Trial Registry in India and the Clinical Trial Registry number- CTRI/2017/03/008139. URL http://ctri.nic.in/Clinicaltrials/regtrial.php?modid=1\&compid= 19\&EncHid=70845.17209. Date of registration- 20/03/2017.

Keywords: Stepped wedge cluster randomised controlled trial, Community-led intervention, Community mental health, Low-and-middle-income countries, Evidence-based practice, Common mental disorders

\section{Background}

Mental illness is a substantial public health burden in India, affecting $10.6 \%$ of the population [1]. There is a shortage of mental health professionals to address the mental health needs of the population, particularly in rural areas [1]. This is further compounded by a high level of public stigma towards mental illness and the lack of accessible mental health care, resulting in a treatment gap of nearly $80-90 \%$ for mental illness in India [1].

In most low- and middle-income countries (LMICs), service delivery models have focused on task-sharing, the process of sharing mental health care tasks with less specialised health workers, such as community health workers [2]. Several programs have been developed to build the capacity of community members and primary care health workers, with the aim of increasing their uptake of mental health tasks and enable access to mental health supports in rural areas [3, 4]. A number of studies in India and other parts of South Asia have shown the efficacy of task-sharing initiatives [5-8].

A possible reason for the inability to scale-up tasksharing models may be that public health systems in low-and-middle-income countries (LMICs) are overburdened with addressing other health needs, and there are limited time and energy to devote to mental health [9-12]. Task sharing approaches may, therefore, need to build community capacity to provide mental health care that complements service provision efforts within the public health system. Atmiyata is a community-led intervention [13] using non-specialised community volunteers for identification, support, and referral for persons with common and severe mental disorders. Atmiyata was previously piloted in 41 villages in the Nashik district of the state of Maharashtra from 2013 to 2015 [13].

As a mental health program, Atmiyata aims to: (i) reduce the treatment gap for common and severe mental disorders; (ii) improve mental health outcomes for people with common mental disorders (CMD); (iii) improve quality of life among people with mental health problems; (iv) improve access to social welfare schemes for people with mental health problems. We hypothesize that the intervention will result in symptomatic improvement in CMD (depression and anxiety) and well-being as well as narrow the mental health 'care' gap [14].
Evaluating both the efficiency as well as the implementation process of this intervention will generate valuable lessons as to how we might sustain the intervention's impact when delivered to a large population.

\section{Methods \\ Design}

We employ a Stepped Wedge Cluster Randomized Controlled Trial (SW-CRCT), with a nested health economic evaluation to assess the impact on persons with CMD and return on investment (ROI) of the intervention in the Indian state of Gujarat. The stepped wedge design is chosen for evaluating the scale-up of Atmiyata intervention as it allows for random allocation of the timing in which clusters receive the intervention [15]. All clusters receive the intervention before the trial ends which is ethically appropriate. The design also allows for Atmiyata intervention to be delivered in a staggered manner to account for practical logistics constraints; it is not feasible to deliver the intervention in all clusters (villages served under groups of primary health centers) simultaneously. Stepwise implementation allows the implementation team enough preparation time and is an efficient use of implementation team resources. Additionally, the staggered implementation of the intervention over time periods allows for more in-depth statistical analysis compared to a pre-post, parallel-arm cluster randomized controlled trial design.

There are 56 primary health centers (PHCs) in the Mehsana district (where the study takes place), and each PHC serves discrete villages within a geographical area. Each village in the geographical area served by a PHC is a cluster in this study. We created 4 groups of clusters (A, B, C, D), each made up of villages covered by 14 PHCs. Groups are created according to geographical location to help reduce the probability of contamination between groups; villages in Group A are farther from Group B villages and villages in Group $C$ are farther from Group D. All groups (A, B, C, D) are allocated to intervention condition at different steps. A 'Step' is the order in which a group of clusters switches from control to intervention condition. On the other hand, 'Period' is defined as a group of observations by the time of measurement. The duration of each period is 5 months to 
accommodate for baseline and 3 months of follow-up data collection (Fig. 1).

This study uses a repeated cross-sectional design with outcome data derived from different participants in each period. All four clusters start at baseline in the control condition and are exposed to the intervention at a regular period of five months (Fig. 1).

\section{Setting}

The intervention is implemented in Mehsana district, located in Western India in the state of Gujarat. It is primarily a rural district (75\% rural), with a rural population of 1.52 million people, of which approximately one million are above 18 years of age. The district is divided into 10 blocks/ sub-districts with a total of

\begin{tabular}{|c|c|c|c|c|c|}
\hline TIMEPOINT & $\begin{array}{l}\text { Period } 1 \\
\text { 1-5months }\end{array}$ & $\begin{array}{l}\text { Period } 2 \\
6-10 \text { months }\end{array}$ & $\begin{array}{l}\text { Period } 3 \\
11-15 \text { months }\end{array}$ & $\begin{array}{l}\text { Period } 4 \\
16-20 \text { months }\end{array}$ & $\begin{array}{l}\text { Period } 5 \\
21-25 \text { months }\end{array}$ \\
\hline \multicolumn{6}{|l|}{ ENROLMENT: } \\
\hline Eligibility screen & $\checkmark$ & $\checkmark$ & $\checkmark$ & $\checkmark$ & $\checkmark$ \\
\hline Informed consent & $\checkmark$ & $\checkmark$ & $\checkmark$ & $\checkmark$ & $\checkmark$ \\
\hline Baseline & $\checkmark$ & $\checkmark$ & $\checkmark$ & $\checkmark$ & $\checkmark$ \\
\hline Allocation & $\checkmark$ & $\checkmark$ & $\checkmark$ & $\checkmark$ & $\checkmark$ \\
\hline \multicolumn{6}{|l|}{ Study phase } \\
\hline $\begin{array}{l}\text { Cluster A (villages } \\
\text { under } 14 P H C s \text { ) }\end{array}$ & $\begin{array}{l}\text { Control } \\
(n=56)\end{array}$ & $\begin{array}{l}\text { Intervention } \\
\qquad(\mathrm{n}=56)\end{array}$ & $\begin{array}{l}\text { Intervention } \\
\qquad(n=56)\end{array}$ & $\begin{array}{l}\text { Intervention } \\
\qquad(n=56)\end{array}$ & $\begin{array}{l}\text { Intervention } \\
\qquad(n=56)\end{array}$ \\
\hline $\begin{array}{l}\text { Cluster B (villages } \\
\text { under } 14 P H C s \text { ) }\end{array}$ & $\begin{array}{l}\text { Control } \\
(n=56)\end{array}$ & $\begin{array}{l}\text { Control } \\
(n=56)\end{array}$ & $\begin{array}{l}\text { Intervention } \\
\qquad(n=56)\end{array}$ & $\begin{array}{l}\text { Intervention } \\
\qquad(n=56)\end{array}$ & $\begin{array}{l}\text { Intervention } \\
\qquad(n=56)\end{array}$ \\
\hline $\begin{array}{l}\text { Cluster C (villages } \\
\text { under } 14 P H C \text { ) }\end{array}$ & $\begin{array}{l}\text { Control } \\
(n=56)\end{array}$ & $\begin{array}{l}\text { Control } \\
(n=56)\end{array}$ & $\begin{array}{l}\text { Control } \\
(n=56)\end{array}$ & $\begin{array}{l}\text { Intervention } \\
\qquad(n=56)\end{array}$ & $\begin{array}{l}\text { Intervention } \\
\qquad(n=56)\end{array}$ \\
\hline $\begin{array}{l}\text { Cluster D (villages } \\
\text { under } 14 \mathrm{PHCS} \text { ) }\end{array}$ & $\begin{array}{l}\text { Control } \\
(n=56)\end{array}$ & $\begin{array}{l}\text { Control } \\
(n=56)\end{array}$ & $\begin{array}{l}\text { Control } \\
(n=56)\end{array}$ & $\begin{array}{l}\text { Control } \\
(n=56)\end{array}$ & $\begin{array}{l}\text { Intervention } \\
\qquad(n=56)\end{array}$ \\
\hline ASSESSMENTS: & & & & & \\
\hline Demographic details & $\checkmark$ & $\checkmark$ & $\checkmark$ & $\checkmark$ & $\checkmark$ \\
\hline $\begin{array}{l}\text { General Health } \\
\text { questionnaire }\end{array}$ & $\checkmark$ & $\checkmark$ & $\checkmark$ & $\checkmark$ & $\checkmark$ \\
\hline $\begin{array}{l}\text { Self-Reporting } \\
\text { Questionnaire }\end{array}$ & $\checkmark$ & $\checkmark$ & $\checkmark$ & $\checkmark$ & $\checkmark$ \\
\hline $\begin{array}{l}\text { Social Participation } \\
\text { Scale }\end{array}$ & $\checkmark$ & $\checkmark$ & $\checkmark$ & $\checkmark$ & $\checkmark$ \\
\hline $\begin{array}{l}\text { EURO-5D Quality of } \\
\text { Life }\end{array}$ & $\checkmark$ & $\checkmark$ & $\checkmark$ & $\checkmark$ & $\checkmark$ \\
\hline $\begin{array}{l}\text { WHO-Disability } \\
\text { scale }\end{array}$ & $\checkmark$ & $\checkmark$ & $\checkmark$ & $\checkmark$ & $\checkmark$ \\
\hline $\begin{array}{l}\text { General Anxiety } \\
\text { Disorder Scale }\end{array}$ & $\checkmark$ & $\checkmark$ & $\checkmark$ & $\checkmark$ & $\checkmark$ \\
\hline $\begin{array}{l}\text { Patient Health } \\
\text { Questionnaire }\end{array}$ & $\checkmark$ & $\checkmark$ & $\checkmark$ & $\checkmark$ & $\checkmark$ \\
\hline $\begin{array}{l}\text { Client Satisfaction } \\
\text { Questionnaire }\end{array}$ & - & $\checkmark$ & $\checkmark$ & $\checkmark$ & $\checkmark$ \\
\hline $\begin{array}{l}\text { Economic } \\
\text { Questionnaire }\end{array}$ & $\checkmark$ & $\checkmark$ & $\checkmark$ & $\checkmark$ & $\checkmark$ \\
\hline
\end{tabular}

Fig. 1 SPIRIT figure. Enrollment, allocation, intervention, and assessment were taken place in all periods as this is a cross-sectional SW-CRCT 
645 villages and 316,536 rural households [16]. Almost half (45.4\%) of Mehsana's rural population has a low standard of living as defined by the Standard of Living Index [17]. Most residents (53\%) are employed in the agricultural sector. The rural population of the Mehsana district is economically disadvantaged as agriculture is not always a viable occupation given uncertain climatic events [16]. In terms of health services, Mehsana has 56 PHCs, 11 Community Health Centers and 1 District Hospital staffed by 2 psychiatrists, along with District Mental Health Program (DMHP) which provides additional human resources (such as a psychologist and social worker) for mental health at the community level. Mental health care is primarily delivered by psychiatrists at the District Hospital and the psychiatrists also visit Community Health Centers on a fortnightly basis in rotation, as part of DMHP. The district hospital has inpatient and out-patient services for persons with mental illness, and limited psychosocial support services.

\section{Participants}

The study sample consists of adult community members with CMD (e.g. anxiety and depression) residing in rural villages in the Mehsana district.

\section{Inclusion Criteria}

- Persons aged 18 years or more and less than 65 years of age

- A Score of 3 or above on the General Health

Questionnaire (GHQ-12), indicating a case with CMD

\section{Exclusion criteria}

- Persons who cannot provide informed consent or decline participation in the study

- Persons with a terminal medical condition

- Persons who have suicidal ideation or plans for suicide at the baseline interview

\section{Primary outcome}

The primary outcome is the symptomatic improvement of CMD as measured using a validated Gujarati version of the GHQ-12 [18] from baseline to 3-month and 8month follow-up to evaluate sustained effects of the intervention. The GHQ-12 is a widely used screening tool with reliable sensitivity for assessing CMD [19]. GHQ-12 is a dichotomous 12-item questionnaire with each item rated on a 4-point scale, with possible responses being "less than usual," "no more than usual," "rather more than usual," or "much more than usual." We used a bimodal scoring method, whereby "less than usual" and "no more than usual" is scored as 0 point, and "rather more than usual" and "much more than usual" is scored as 1 [18]. GHQ-12 scores will be analyzed as both continuous (ranging from 0 to 12) and categorical outcomes (case defined as 3 and above scores on GHQ scale; non-case as less than 3 scores on GHQ scale).

\section{Secondary outcomes}

Secondary outcome measures are assessed at 3 and 8 months after the start of the intervention (Table 1).

\section{Quality of life}

Improvement in quality of life among persons with CMD is assessed using a validated Gujarati version of EURO Quality of life- 5D (EQ-5D) [20]. The EQ-5D's descriptive system is a preference-based Health Related Quality of Life measure with one question for each of the five dimensions that include mobility, self-care, usual activities, pain/discomfort, and anxiety/depression measured at 5 levels: no problems, slight problems, moderate problems, severe problems, and extreme problems. A lower score indicates a better quality of life [20].

\section{Psychiatric symptoms}

Improvement in psychiatric symptoms is assessed using a validated Gujarati version of the Self Reporting Questionnaire (SRQ). SRQ is a scale developed by the World Health Organization to screen for psychiatric disturbances for low- and middle-income countries. It consists of 20 questions which are scored $1=$ yes and $0=$ no, indicating the presence or absence of a particular symptom over the past month. SRQ is a continuous scale and responses are calculated as a total score ranging from 0 to 20, with lower scores indicating recovery of symptoms [21].

\section{Disability}

Reduction of disability and reduction in a number of days unable to work and improvement in productivity is assessed using a validated Gujarati version of the World Health Organization's Disability Assessment Scale (WHO-DAS-12). The WHO-DAS-12 assesses overall functioning, in relation to difficulties due to health conditions. The scale has 12 items, with a 5-point rating scale ranging from none, mild, moderate to severe and extreme. Responses are calculated as a total score ranging from 12 to 60 [22].

\section{Depression and anxiety symptoms}

Improvements in depression symptoms are assessed using a validated Gujarati version of the Patient Health Questionnaire (PHQ-9). PHQ-9 scores range from 0 to 27 , with a higher score indicating more severe symptoms [23]. Improvements in anxiety scores are assessed by using a validated Gujarati version of Generalized Anxiety 
Table 1 Follow-up assessment of tools with time points

\begin{tabular}{llll}
\hline ASSESSMENT TOOLS & Baseline & 3-month follow-up & 8-month follow-up \\
\hline Demographic information & $\checkmark$ & $\checkmark$ & $\checkmark$ \\
General Health questionnaire & $\checkmark$ & $\checkmark$ & $\checkmark$ \\
Self-Reporting Questionnaire & $\checkmark$ & $\checkmark$ & $\checkmark$ \\
Social Participation Scale & $\checkmark$ & $\checkmark$ & $\checkmark$ \\
EURO-5D Quality of Life & $\checkmark$ & $\checkmark$ & $\checkmark$ \\
WHO-Disability scale & $\checkmark$ & $\checkmark$ & $\checkmark$ \\
General Anxiety Disorder Scale & $\checkmark$ & - & $\checkmark$ \\
Patient Health Questionnaire & $\checkmark$ & $\checkmark$ & $\checkmark$
\end{tabular}

Disorder (GAD-7) with total scores ranging from 0 to 21 [24].

\section{Social Participation}

Increases in social participation is assessed using the Social Participation Scale (SPS) [25]. The SPS is an 18item interview-based instrument measuring perceived problems in major domains of life such as learning and applying knowledge, communication, mobility, self-care, domestic life, interpersonal interactions, major life areas (like work, life, and employment) and community (like leisure, recreation, political life). The scale allows quantification of participation restrictions experienced by people affected by disability or other stigmatized conditions. The 18 items are rated on a 5-point scale ranging from 0 (no problem) to 5 (large problem). Responses are calculated as a total score ranging from 0 to 90 [26].

\section{Service User Satisfaction}

User-satisfaction with the intervention is assessed using a validated Gujarati version of the Client Satisfaction Questionnaire (CSQ) [25] at 8-month follow-up. The CSQ is an 8-item scale assessing client satisfaction with care/treatment received. The scoring uses a 4-point rating scale and is scored by summing the individual scores to produce a range of 8 to 32, with higher scores indicating greater satisfaction with care [27].

\section{Specificity of identification of CMD cases}

The accuracy of Champions identifying community members with CMD by Champions is assessed using the GHQ-12.

\section{Economic Evaluation}

The most common measure of efficiency of interventions in the health sector is cost-effectiveness analysis (CEA) which measures health-related benefits and expresses these in a natural unit such as lives saved, or symptoms reduced. A return-on-investment analysis, however, expresses benefits in monetary terms relative to investments made. Expressing both the costs and the full range of benefits of an intervention in the same units (money) has the distinct advantage of making investment decisions very straightforward [28]. If the money value of the benefits of an intervention is larger than the cost of the intervention, it may be regarded as a sound investment. Hence, we have chosen to do ROI analysis for the Atmiyata intervention.

Costs are calculated using both government and societal perspective [29]. Perspective determines the cost components to be included in any cost analysis and societal perspective is the broadest viewpoint that covers all costs irrespective of who incurs these costs. On the other hand, a government perspective only includes costs incurred by the government for a particular health intervention. Costs are accounted under two categories: 1 . total cost of the intervention and; 2 . treatment cost of CMDs. Treatment costs are categorized as: direct medical cost, direct nonmedical cost, and indirect costs. Direct medical costs include out of pocket expenses incurred in order to seek treatment (e.g. diagnostic tests, fees for consultation in clinics, traditional healers, hospitals, bed day charges at a public or private health facility). Direct non-medical costs include the amount spent for travelling to the health facility for the patient and accompanied persons for treatment, the amount spent on meal/food taken while waiting for treatment, expenses for overnight accommodation for seeking care, etc. Indirect costs represent the opportunity cost for the patient and their household members' time related to CMD. Cost data is collected at baseline, 3 months and 8 months from all study participants. Time spent by the champion will be obtained from the program implementation data. The minimum wage rate of Gujarat will be used to value their time. Total hours spent on the program will be multiplied by hourly wage (obtained from the minimum wage rate) to get the time cost of the Champions. Benefits are considered in terms of improved health, functioning, participation, productivity, increased 
saving and investment, reduced informal care giving and health and welfare services. Lost workdays before and after the intervention will be obtained through the WHO-DAS 12 questionnaire and will be linked to the minimum wage rate to estimate the aggregate effect. Being alive and healthy is also considered valuable and the overall value of a life year can be broken down into its economic (instrumental) and health (intrinsic) elements. Following the approach used in prior ROI analyses in mental health, we use the figure of 0.5 times the per-capita income of India as the value of a healthy life year [28, 30, 31].

\section{Intervention condition}

The Atmiyata intervention has been described extensively elsewhere [13]. Briefly, Atmiyata is a complex psychosocial intervention involving two-tiers of community volunteers for identification and support to people in distress and with symptoms of common mental disorders. The first tier consists of community volunteers called Atmiyata Mitras who are from different caste and religion-based sections of the village, trained to identify persons in mental distress. The second tier consists of Atmiyata Champions, who are important community members (e.g. former teachers, community leaders) with leadership and communication skills and are well-known and approachable in their village. Champions are trained to identify and provide structured counseling to persons with significant mental distress, including the ones referred by Mitras. Given the social barriers based on caste, gender, religion, the identification and support by Champions and Mitras ensure equitable reach and improves coverage of the intervention across the entire village.

In the Atmiyata pilot intervention in the state of Maharashtra in 2013-2015, Champions and Mitras were trained by the project team. In this study, the Atmiyata Gujarat program, where the target population is substantially larger, Champions are identified and trained by Community Facilitators (CF) who typically have a master's degree in social work or related fields, are locally based and aware of community dynamics. CFs first map their allotted villages, then identify and recruit the Champions, train them and provide ongoing mentoring support to Champions. The CFs are recruited, trained and mentored by Project Managers (PM). Each PM supports 7-8 CFs. Each CF supports 40-50 Champions (1 per 1000 population), and each Champion has 4-5 Mitras. The principal Investigator (PI) monitors and supports the PMs (Fig. 2).

Mitras receive $4 \mathrm{~h}$ of training from a Champion based in a particular village. Champions receive $40 \mathrm{~h}$ of training over 3 weeks, at a central location in the block or village area. PMs and CFs receive $55 \mathrm{~h}$ of training over 5 weeks, with additional 8-h sessions on how to be a master-trainer. The methodology of the training is interactive, reflective and participatory.

The Champions are trained: (i) to identify persons with CMD and provide evidence-based 4-6 counseling

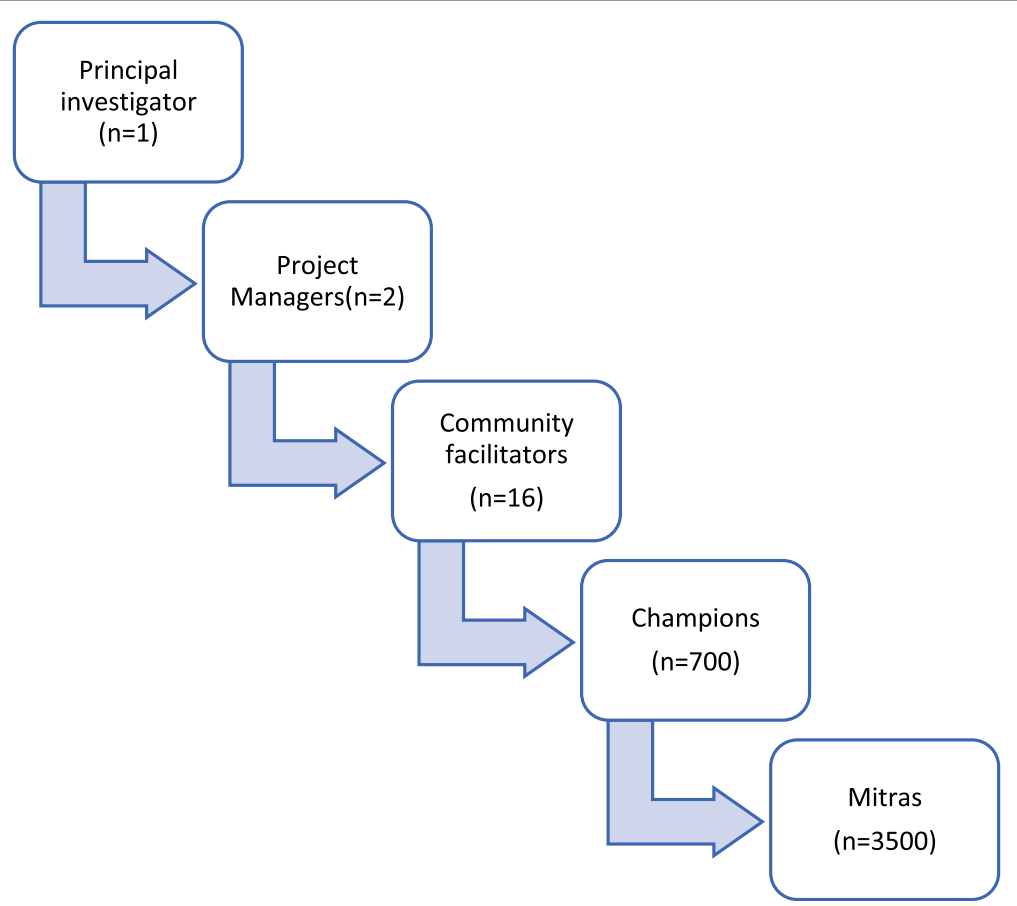

Fig. 2 Atmiyata Implementation team structure 
sessions; (ii) to raise community awareness on social issues by 'narrow-casting' four films; 10-min films dubbed in Gujarati on commonly experienced social issues in the community such as unemployment, family conflict, domestic violence, and alcoholism. Films are developed to build community mental health awareness and were not designed for training purposes or for intervention delivery related to counselling or symptom reduction. The films are shown to community members in the village where the Champions reside, typically in a public space in the village. Champions show these films in a small group of 3 to 4 people at communal meeting places in a village such as a temple, at a farm or Champion's house [32].; Champions also (iii) make referrals of persons with severe mental disorders to mental health services offered within the public health system when required, and (iv) enable access to social benefits for persons with mental health problems, such as government schemes for paid work opportunities.

Counseling sessions by Champions are based on three evidence-based techniques used in prior and similar tasksharing programs [33, 34]: active listening, activity scheduling and problem-solving techniques. The training includes basic skills of empathy, non-judgemental behaviour, rapport building, verbal and non-verbal communication and creating a safe environment to build skills for 'active listening'. Activity scheduling techniques enable individuals to explore reasons why they have been avoiding activities and schedule pleasurable and valuable activities to resume day to day routine $[35,36]$. Problem-solving techniques are used for a positive orientation towards the problem which enables viewing problems as solvable, and as opportunities to learn and change. Champions are trained to deliver 4-6 sessions of counseling over a period of 6 to 12 weeks, with no set time between sessions. Each session lasts 20 to 40 min based on mutual agreement between the Champion and the participant. The number of sessions delivered to the participant is also left to mutual agreement between the Champion and the participant. As this is an implementation research study, we aim to assess what happens when the intervention is implemented in 'real-life' settings where the number, duration, and frequency of sessions will vary between the different provider (Champion) and participant dyad. We therefore provide broad guidelines and recommendations on the number, duration and frequency of sessions to Champions (e.g. a range of sessions from 4 to 6 sessions, duration from 20 to $40 \mathrm{~min}$, and period of 6-12 weeks) which both mimics what is likely to happen in reallife clinical settings and allows us to explore the possibility of dose-response effects in the study. Champions deliver the intervention at the participant's home or immediate surroundings at a location preferred by the participant (e.g. champion's home, a community place such as village hall, temple, etc.). Thus, participants do not have to travel to receive the intervention. The delivery of intervention is in the Gujarati language.

Supervision and mentoring are important components of our Intervention [13]. In the Atmiyata intervention, the PI (who is also a clinician) is the primary supervisor of the PMs and CFs. The PMs and CFs supervise and mentor champions delivering the intervention. The PMs and CFs receive 7 days of training in the intervention with an additional 3 days of training on supervision and mentoring. These 10 days of training for CFs and PMs are supplemented through routine supervision discussions with the PI. The PMs and CFs go on to conduct a 7-day training for the Champions. CFs visit the Champions on-site once a month for hands-on mentoring sessions. During these visits, CFs discuss with Champions the counseling sessions delivered by them, check the structure of each session and troubleshoot any difficulties in using counseling techniques. CFs also help champions clarify concepts and if necessary, demonstrate counseling techniques. These monthly visits ensure the quality and fidelity of the intervention are maintained. Day-long refresher sessions are scheduled once every three months to bring Champions and CFs back together to discuss common challenges and strategies to overcome challenges in delivering the intervention.

\section{Adverse events}

We considered adverse events as attempted suicide, selfharm or death by suicide. A protocol for reporting and recording of adverse events is provided in the Additional file 1. All team members are trained by the data manager and PI on proper identification, recording, and reporting of adverse events. All adverse events are tracked as per the protocol and reported to the Institutional Ethics Committee within 15 days of occurrence. We decided to only track the above-mentioned adverse events because Atmiyata provides low-intensity counseling package delivered by Champions to people with distress and CMD, and other events such as non-suicide related hospitalizations are not directly related to the service being provided. This specific definition of adverse events was agreed upon by the ethics committee advising the project on ethical considerations and documented and defined in the trial protocol.

\section{Comparison condition}

Participants in the control condition receive Enhanced Usual Care (EUC). EUC is offered to all participants in the comparison condition who scored 3 and above on GHQ-12. EUC provides information on the impact of distress on their physical and mental health and relevant information of accessible and available public mental health care services, including services by the District Mental Health Programme (DMHP), and helplines for 
mental health support and domestic violence in and around Mehsana district.

The EUC conditions also has provisions for providing active support to participants in crisis. A crisis is defined as the participant revealing a recent self-harm attempt or expressing thoughts of self- harm during data collection. Such participants are encouraged to seek help immediately and the data collectors seek participant consent to inform their family member or a friend about the crisis and thus, mobilize social support to deal with the crisis.

\section{Sample size and power calculations}

A trained lay health worker-led intervention study conducted in India reported a risk difference of $12 \%$ at follow up between intervention and control condition for the recovery of CMD patients [5, 37]. The sample size for this SW-CRCT is calculated to detect a 13\% difference in CMD cases at a 3-month follow-up using GHQ12 as a categorical measure between the intervention (58\% improved) and control condition (45\% improved). Assuming an intra-cluster correlation coefficient of ICC $=0.1$, number of steps $(t=4)$, number of clusters randomized in each step $(\mathrm{k}=14)$, average cluster size $(m=4)$, power $(80 \%)$ and alpha of 0.05 , a sample size of 1120 participants is needed, with approximately 56 individuals per cluster per period (Fig. 1,3). The sample size was calculated using the "stepped wedge" function of STATA version 14 [38].

\section{Randomization and treatment allocation}

The unit of randomization in stepped wedge trials is a cluster or group of clusters, allocated to different steps. In India, PHCs serve discrete villages and hence we have used the PHC as a unit to identify discrete geographical areas consisting of all villages under a PHC. This geographical area is taken as a cluster for our study. Typically, a PHC in Gujarat covers a population of $25,000-30,000$ across 12-13 villages. There are 56 PHCs in the Mehsana district, split into 4 groups (A, B, C, D), with each group consisting of all villages under 14 PHCs. These groups of clusters (A, B, C, D) are sequentially allocated to different steps at given time periods. Randomization takes place at the level of participants, all the participants are randomly selected for each period from each of these groups of clusters $(\mathrm{A}, \mathrm{B}, \mathrm{C}, \mathrm{D})$ as described in the recruitment section below. The administrative organization of primary health care in Gujarat ensures that the inter-PHC movement of people for health care is minimal and avoids contamination of the intervention. Since double-blinding is not possible in such psychosocial intervention trials, several other procedures are used to minimize contamination and bias. To minimize contamination due to the intervention and control village members meeting and potentially discussing the intervention, clusters are geographically dispersed.
Second, the data collection team is separate from the intervention team and blind to the treatment status. Third, the data collection staff receives initial training and re-training at repeated intervals to ensure the quality of data collected.

\section{Recruitment}

In the control condition (enhanced usual care), a screening list is generated from the district electoral roll using a systematic random sampling method with pre-decided random start and random interval, with every $n$th number from the pool being selected. We used electoral roll as it is the most complete, comprehensive and accessible national frame of residential addresses in India and electoral rolls are extensively used for drawing a random sample from the general population [39]. For each group of clusters $(\mathrm{A}, \mathrm{B}, \mathrm{C}$, and $\mathrm{D})$ electoral rolls from all the villages under $14 \mathrm{PHCs}$ are included. Since the prevalence of CMD is $4-8 \%$, according to the National Mental Health Survey, India [1], a screening list using a minimum prevalence of $4 \%$ and assuming $25 \%$ missing persons is prepared. For each group (A, B, C and D) in each period of the control condition, a screening list of 1800 participants is created and screened (GHQ-12 score of 3 and above) to achieve the target sample of 56 participants.

A different recruitment procedure is used in the intervention condition, as using structured questionnaires for identification (e.g. GHQ, PHQ) was perceived as impractical when implementing the intervention at scale and seen as stigmatizing in a community setting. Champions are trained to identify a person in their catchment area (i.e. villages) with CMD based on symptoms described by the participant during an unstructured interview. When a Champion identifies a person with CMD to whom they intend to provide 4-6 counseling sessions to, they are asked to inform their CF who in turn informs the data manager who creates a caseload list for each Champion. All caseload lists across Champions are then merged to create a master list. The master caseload list has the residential address of the persons identified by the Champion, who are then approached by the data collection team. Champions obtained verbal consent from each person before providing any personal information such as the address to the CF. CFs are trained to maintain confidentiality of this information (see the section on data storage, confidentiality below). The sample for the intervention condition is drawn from this master caseload list using a computer-generated random method. The drawn sample is screened by data collection staff using GHQ-12 (score of 3 and above) for recruiting intervention participants. This screening and subsequent baseline data collection for participants meeting the inclusion criteria is done prior to the Champion starting psychosocial counseling sessions with the 


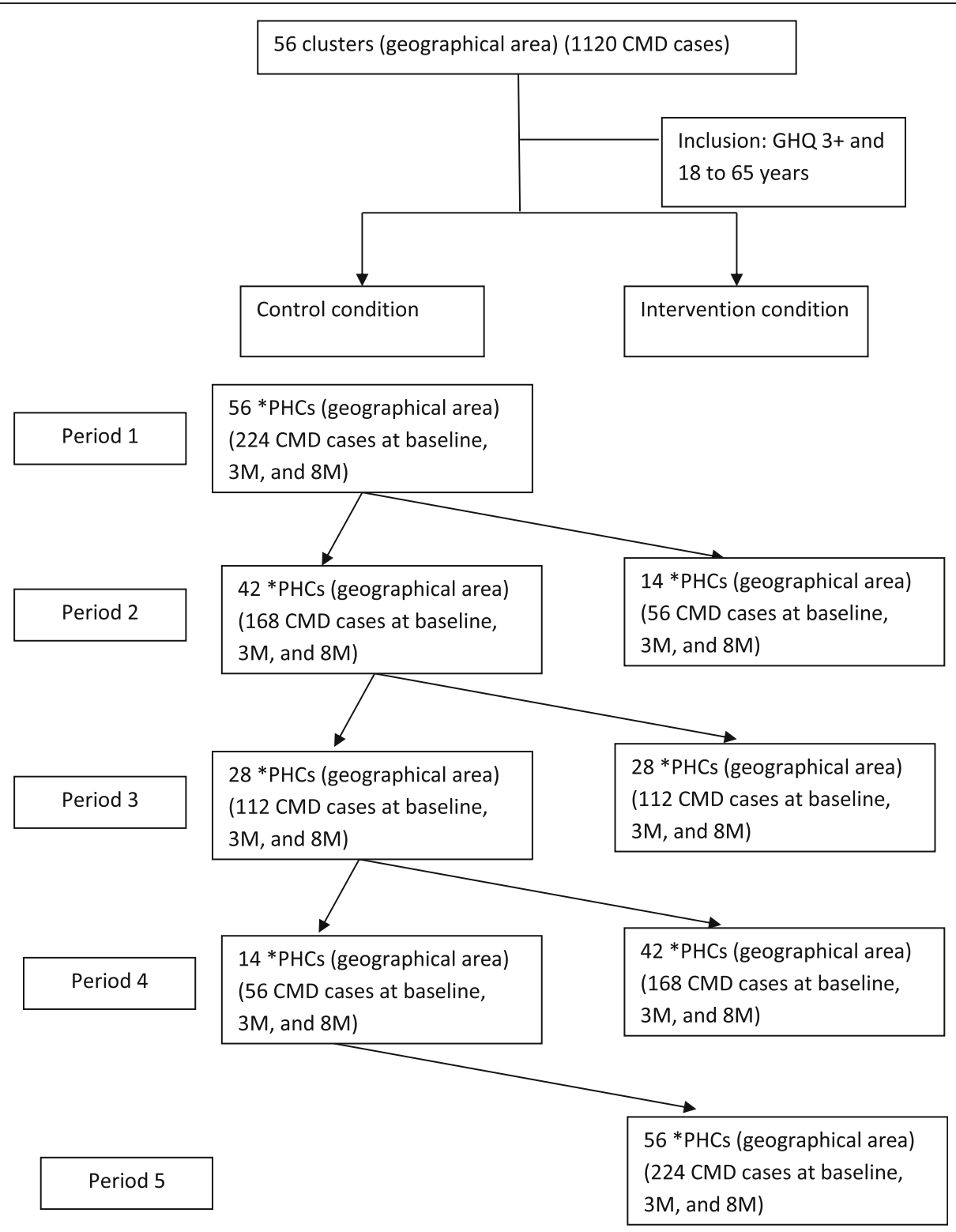

Fig. 3 CONSORT Flow chart for ATMIYATA SW-CRCT design

*PHCs in the consort diagram refers to groups of clusters based on geographical area

participant. This process is continued until the target sample size of 56 cases is reached for each cluster and period. A different recruitment procedure for the intervention condition was chosen in order to estimate the specificity (accuracy) of ident ification of CMD cases by Champions, which is one of the secondary outcomes. Using similar recruitment procedures in both control and intervention condition (using electoral rolls) would have answered the coverage question (how many people with CMD in the community were identified by the Champion) but not allow us to assess accuracy of identification. We first want to establish whether Champions are accurately able to identify persons with $\mathrm{CMD}$, before addressing the coverage question. Furthermore, we have other data to estimate the population coverage of the intervention.

\section{Data collection}

Written Informed consent is sought from all participants. A thumb impression and signature of a witness is taken for illiterate participants [40]. Data is collected by trained researchers in two stages using a paper-pencil method. In the first stage, demographic data along with GHQ-12 is collected. The data collectors score the 
GHQ-12 using a scoring sheet and if the participant has a score of 3 or more, secondary outcome data is collected at the same time. The questionnaire for secondary outcome data takes 40-45 min to complete. Participants are not compensated for their time, as this is a volunteer-led intervention. Data collection staff travel to participant's home for data collection to avoid any travel costs for participants. Data collectors are recruited from the intervention district but recruited from different villages. Data collectors are not paired (based on age, gender, caste, and religion) with participants and they are not assigned participants from their villages for data collection, thus reducing the likelihood of study staff having prior acquaintance with the participants. Furthermore, if any study staff had any prior personal acquaintance with a participant, they were replaced with another data collector who was not acquainted with the participant.

\section{Data management}

Atmiyata uses a comprehensive data management system that aids in collecting high-quality data by maintaining on-going- on-site and off-site quality assurance and quality control checks. Research staff (data collectors) handling data are thoroughly trained in interview techniques and procedures for sensitive data handling. Several measures to control for quality of data collected are implemented, including weekly checks, field monitoring visits twice a month by data manager and spot checks (once a month). Additionally, refresher training sessions are provided once in 4 months for quality assurance purposes. The data manager ensures completeness and legibility of the data prior to data entry and is responsible for storing all the data. A designated data entry person is trained for specific entry guidelines to avoid erroneous data entry. The de-identified data is entered in a password-protected Excel sheet. Personally identifiable information is not entered in the database. Raw data is not uploaded on the internet; instead all entered data is shared with the statistician through offline electronic data transfer from the site by the project manager on a monthly basis. The statistician collates the data, maintains the database, and reviews data quality in terms of numbers, consistency and completeness. Measurement of percentage agreement among the data collectors is obtained once a year, to ensure the reliability of the data collected. Several strategies are adopted to achieve adequate participant enrolment including three telephonic follow-up calls to participants not available during inperson visits, two reminders for follow-up visits and rescheduling visits as per participant convenience. Recruitment, follow-up rates, and missing data are discussed at monthly team review meetings between a data manager and data collectors.

\section{Data storage, security, and confidentiality}

Study data is anonymized using unique study identification codes for participants, which is matched to the physical consent form and then entered in the study database. Only the consent form includes personally identifiable details. A code sheet linking the participant's personal identifiable information is linked to the unique study identification code. Data is stored on a passwordprotected external hard drive periodically as a back-up. All consent forms and data forms are stored in a locked cabinet at the site office in Mehsana, accessible only to the PI and data manager. After the study is over, the data will be stored in the sealed cabinet as required by Indian regulations.

\section{Data monitoring}

An advisory committee consisting of 4 experts in medical ethics, public health administration and public health and social science research was formed to monitor the implementation and research. The Committee meets every 6 months with the research team and makes periodic site visits to personally interact with a few participants. All adverse events will be reported to the committee.

\section{Statistical analysis}

Baseline characteristics will be summarized using counts (percentages) for categorical variables and means (SD) for continuous variables. The analysis will be based on intention to treat and participants will be analyzed in the group that the cluster was assigned to at each time point.

The analysis plan is based on the Hussey and Hughes model for the analysis of cross-sectional SW-CRCT designs [15]. Generalized Linear Mixed Model (GLMM) will be used to determine the size and direction of the difference between the control and intervention conditions for primary and secondary outcomes. The estimated intervention effect will be reported as the mean outcome difference for continuous variables and Odds Ratio for categorical variables between intervention and control condition assuming a constant treatment effect over time. Estimates of the difference and 95\% CIs will be calculated. To take the time effect into account, all analyses will be adjusted for time (periods) of the intervention and clusters. Period (time) and intervention (counseling sessions) will be specified as fixed effects and clusters as a random effect. The analysis will be adjusted for baseline covariates to account for potential imbalance arising due to different recruitment procedures and regional differences across control and intervention conditions. The analysis plan does not include any interim analyses.

Two broad model extensions [41], random cluster by period effect and random cluster by treatment effect will 
be used for secondary analysis. The secondary analysis will investigate an interaction effect between intervention and time and the interaction effect between cluster and time. Additional analyses of the primary outcome will be conducted controlling for demographic variables if required. Statistical analyses will be carried out using STATA version 14 [38].

\section{Economic evaluation analysis}

All data will be analysed in Microsoft excel. The ratio of costs and benefits will be calculated and will be presented as an ROI. This will inform whether Atmiyata intervention is a sound investment. Apart from this, the study will also provide information on the economic burden of CMD in the Mehsana district, which is of value to funders, policymakers and can be used for advocacy purposes. The economic burden of CMD will include direct medical cost (out of pocket expenses on drugs, diagnostics, consultations fees during outpatient visits and hospitalization), direct non-medical cost (out of pocket expenses on transport, food, accommodation while seeking treatment) and indirect cost (time spent by a patient and accompanied persons). As one of the secondary outcomes of the study is to understand the changes in the quality of life of the CMD patient using the EQ-5D, we will conduct a cost-utility analysis as an additional analysis in the economic evaluation. Costutility analysis expresses the value for money in terms of a multi-dimensional health outcome. The incremental cost-effectiveness ratio, in this case, is usually expressed as the incremental cost to gain an extra quality-adjusted life-year (QALY) [29]. Consolidated Health Economic Evaluation Reporting Standards (CHEERS) checklist will be followed while reporting the results of ROI and costutility analysis [42].

\section{Discussion}

Atmiyata is a community-led intervention focused on reducing distress, particularly depression and anxiety symptoms in rural communities in India. The evaluation of the intervention through SW-CRCT, offers several unique opportunities. There is limited literature on SWCRCTs conducted in low and middle-income countries, particularly involving mental health interventions. This study will contribute to this sparse evidence base and be able to use implementation lessons to inform further scale-up of the intervention to other districts and states, as well as to inform potential intervention scale-up in other settings.

Several challenges exist with using an SW-CRCT design, including a lack of consensus on the model of analysis [41]. Although the Ottawa Statement [43] and the Council for International Organizations of Medical
Sciences (CIOMS) guidelines [44] provide relevant guidance to the design and conduct of Cluster Randomised Trials, neither document provides guidance specific to SW-CRCT. Other analytical challenges include modelling secular trends, time-varying intervention effect and modelling treatment effect heterogeneity. Different recruitment strategies may confound the statistical analysis. For intervention condition, the sample is drawn from cases identified by Champions. Champions are trained to identify people with CMD, there is a likelihood that they may identify people with higher GHQ scores, and this may confound the analysis and effect. We acknowledge this confounding effect due to different recruitment strategies, and the statistical analysis plan includes adjustment with baseline covariates to account for this confounding. Despite these limitations, we chose this design as being ethical and equitable, as all clusters receive intervention before the end of the trial. In addition, with a control and an intervention period in each cluster, outcomes can be compared within and across clusters which increase statistical power.

Another challenge is the lack of consensus on the cutoff score for depression using GHQ-12. Patel et al. [45] recommended a cutoff score of 7/8 for clinic-based populations. Goldberg et al. [46] suggested the best threshold for GHQ-12 scores varied from 1/2 to 6/7, with the most common cut-off score being 2/3. A recent Indian study confirmed an optimal cut-off score of 2 for community studies based on a receiver operating characteristic curve (ROC) analysis [47]. Taking all the above into account, we conservatively chose 3 as the cutoff score for our community study as it ensures the inclusion of community participants with mild to moderate CMD.

We anticipate challenges during data collection of the trial in a large community setting. Due to stigma and silence around mental ill health, there is a likely reluctance to participate in community mental health studies. Refusal to participate may also be due to the caste, gender, religion, and other social attributes. To address these challenges, data collectors are trained and retrained on how to build rapport with participants and to maintain privacy and confidentiality. We recruited data collectors with diverse social attributes to represent Mehsana's heterogeneous population.

Community members often change residence, or the address is incorrectly entered in the electoral register, which increases the time for identification of control condition participants. Data collection timelines also have to accommodate for community events such as farming season, religious festivities. Safety of data collection staff is an equally important concern given the large geographical area being covered. The team will conduct regular meetings to troubleshoot challenges on the field to adhere to the protocol. 
Another challenge is to assess the extent to which Champions adhere to the counselling model that they have been trained in. A qualitative method is employed in to assess the fidelity. To do this, we will analyze audio recordings of counselling sessions, assessing a random sample of recordings amounting to $5 \%$ of the total counselling sessions delivered by Champions and the results of which will be published separately.

\section{Trial status}

Protocol version: v4, 07 May 2018

Protocol modification in Clinical Trial Registry- 06 April 2018

Date of recruitment- April 2017

Date of recruitment completion- August 2019

We intend to publish trial results in an open-access journal and through meetings with various district and state-level stakeholders.

\section{Supplementary information}

Supplementary information accompanies this paper at https://doi.org/10. 1186/s13063-020-4133-6

Additional file 1. Protocol for Tracking Adverse events.

Additional file 2. Gatekeeper consent form.

Additional file 3. Consent form for research participant.

\section{Abbreviations}

CF: Community Facilitator; CMD: Common mental disorders; CSQ: Client Satisfaction Questionnaire; DMHP: District Mental Health Programme; EQ5D: EURO Quality of life- 5D; EUC: Enhanced Usual Care; GAD-7: Generalized Anxiety Disorder 7; GHQ-12: General Health Questionnaire 12; PHC: Primary Health Centre; PHQ-9: Patient Health Questionnaire 9; PI: Principal Investigator; PM: Project Manager; ROI: Return on Investment; SPS: Social Participation Scale; SRQ-20: Self Reporting Questionnaire 20; SWCRCT: Stepped Wedge Cluster Randomized Controlled Trial; WHO-DAS12: World Health Organization's Disability Assessment Scale 12

\section{Acknowledgements}

We would like to gratefully acknowledge the Department of Health and Family Welfare, Gujarat and our mentor committee members for their collaboration and support.

\section{Authors' contributions}

$\mathrm{JK}, \mathrm{KJ}$, and DP drafted the first version of the manuscript, SC reviewed the section on economic evaluation, SP and LSZ provided additional inputs by editing the draft and adding to the introduction, methods and discussion section. SK provided feedback for the intervention section and references. All authors read and approved the manuscript.

\section{Funding}

Grand Challenges Canada (Grant no- 0792-05) and Mariwala Health Foundation, India jointly funded the trial. Grand Challenges Canada scientific committee reviewed study design, analysis, and interpretation of the data. The funder has not contributed to manuscript writing. Contact details- Grand Challenges Canada- Patrick.coburn@grandchallenges.ca Mariwala Health Foundation-rm@mariwalahealthinitiative.org

\section{Availability of data and materials}

The datasets will be available to appropriate academic parties on request from the principal investigator in accordance with the data sharing policies of the institute within one year of completion of a complete analysis of the data.

\section{Ethics approval and consent to participate}

Permission has been obtained from the Department of Health and Family Welfare, Government of Gujarat for project implementation and data collection. Indian Law Society's Ethics committee approved the study (ILS/ 14/2017) and an additional ethical approval was obtained from the local ethics committee from Hospital for Mental health, Ahmedabad. The trial is registered prospectively with the clinical trial registry, India and the Clinical Trial Registry number- CTRI/2017/03/008139.

Before approaching individual participants, data collection staff informs the head of the village council about the study and the purpose of the data collection. Written informed consent (or thumb impression with a signature of witness in case of illiterate participants) is obtained from each participant enrolled in the trial after providing information about the study including the purpose of the study, benefits, and risks for the participant and information about withdrawal from the study. A copy of informed consent is given to each research participant. Gatekeeper consent and Participant consent forms are uploaded as Additional files 2 and 3 .

\section{Consent for publication}

Not applicable.

\section{Competing interests}

The authors declare that they have no competing interests.

\section{Author details}

'Centre for Mental Health Law and Policy, Indian Law Society, Law College Road, Pune 411004, India. ${ }^{2}$ George Institute for Global Health, Elegance Tower, 311-312, Third Floor, JasolaVihar, New Delhi, Delhi 110025, India. ${ }^{3}$ Netherlands Institute for Mental health and Addiction (Trimbos Institute), Da Costakade 45, 3521 VT Utrecht, the Netherlands.

Received: 6 June 2019 Accepted: 1 February 2020

Published online: 21 February 2020

\section{References}

1. Gururaj G, Varghese M, Benegal V, Rao GN, Pathak K, Singh LK, et al. National Mental Health Survey of India, 2015-16. Bengaluru: National Institute of Mental Health and Neuro Sciences, NIMHANS Publication No 128; 2016.

2. Hoeft TJ, Fortney JC, Patel V, Unützer J. Task-Sharing Approaches to Improve Mental Health Care in Rural and Other Low-Resource settings: A Systematic Review. J Rural Health. 2018;34(1):48-62. https://doi.org/10.1111/jrh.12229 Epub 2017 Jan 13

3. Patel V, Araya R, Chatterjee S, Chisholm D, Cohen A, De Silva M. Treatment and prevention of mental disorders in low-income and middle-income countries. Lancet. 2007:370:991-1005.

4. Balaji M, Chatterjee S, Koschorke M, Rangaswamy T, Chavan A, Dabholkar H, et al. The development of a lay health worker delivered collaborative community based intervention for people with schizophrenia in India. BMC Health Serv Res. 2012;12:42.

5. Patel V, Weiss H, Chowdhary N, Naik S, Pednekar S, Chatterjee S, et al. Effectiveness of an intervention led by lay health counsellors for depressive and anxiety disorders in primary care in Goa, India (MANAS): a cluster randomised controlled trial. Lancet. 2010;376:2086-95.

6. Chatterjee S, Naik S, John S, Dabholkar H, Balaji M, Koschorke M, et al. Effectiveness of a community-based intervention for people with schizophrenia and their caregivers in India (COPSI): A randomised controlled trial. Lancet. 2014;383:1385-94.

7. Chowdhary N, Anand A, Dimidjian S, Shinde S, Weobong B, Balaji M, Patel V. The Healthy Activity Program lay counsellor delivered treatment for severe depression in India: Systematic development and randomised evaluation. $\mathrm{Br}$ J Psychiatry. 2016;208(4):381-8. https://doi.org/10.1192/bjp.bp.114.161075.

8. van Ginneken N, Tharyan P, Lewin S, Rao GN, Romeo R, Patel V. Nonspecialist health worker interventions for mental health care in low- and middle- income countries. Cochrane Database Syst Rev. 2011; [cited 2013 Nov 19] Available from: https://www.ncbi.nlm.nih.gov/pubmed/24249541.

9. National Institute of Mental Health and Neurosciences. Report of Evaluation of the District Mental Health Programme. Bangalore: National Institute of Mental Health and Neurosciences; 2003

10. Kumar A. District Mental Health Programme in India: A Case Study. J Heal Dev. 2005;1:24-35. 
11. Murthy R. District Mental Health Programme (DMHP) - Summary report of Chandigarh. Haryana: Himachal Pradesh and Punjab states; 2003. p. 1-20.

12. Indian Council for Market Research. Evaluation of the District Mental Health Programme. Final Report submitted to Ministry of Health and Family Welfare. Delhi: Ministry of Health and family welfare; 2011.

13. Shields-Zeeman L, Pathare S, Walters BH, Kapadia-Kundu N, Joag K. Promoting wellbeing and improving access to mental health care through community champions in rural India: the Atmiyata intervention approach. Int J Ment Heal Syst. 2017;11:6. https://doi.org/10.1186/s13033-016-0113-3.

14. Pathare S, Brazinova A, Levav I. Care gap: A comprehensive measure to quantify unmet needs in mental health. Epidemiol Psychiatr Sci. 2018;27(5) https://doi.org/10.1017/\$2045796018000100 Available from: https://www. cambridge.org/core/product/identifier/S2045796018000100/type/journal_ article.

15. Hussey MA, Hughes JP. Design and analysis of stepped wedge cluster randomized trials. Contemp Clin Trials. 2007;28(2):182-91.

16. Census of India 2011- District Census handbook- http://www.censusindia. gov.in/2011 census/dchb/DCHB.html

17. Gujarat Social Infrastructure Development Society (GSIDS), General Administration Department (Planning), Government of Gujarat. District Human Development Report - Mehsana. UNDP Report. Gandhinagar;2016. https://www.in.undp.org/content/dam/india/docs/human-development/ District\%20HDRs/16.\%20Mahesana_DHDR_2017.pdf.

18. Goldberg D, Williams P. A User's Guide to the General Health Questionnaire (GHQ). London: GL assessment; 1988.

19. Goldberg D. GHQ and psychiatric case. Br J Psychiatry. 1979;134:446-7.

20. Herdman M, Gudex C, Lloyd A, Janssen MF, Kind P, Parkin D, et al. Development and preliminary testing of the new five-level version of EQ-5D (EQ-5D-5L). Qual Life Res. 2011;20(10):1727-36. https://doi.org/10.1007/ s11136-011-9903-X.

21. World Health Organisation. A User's Guide to the self reporting questionnaire (SRQ). 1994. Retrieved from http://apps.who.int/iris/ bitstream/10665/61113/1/WHO_MNH_PSF_94.8.pdf

22. World Health Organisation. Measuring Health and Disability: Manual for WHO Disability Assessment Schedule (WHODAS 2.0). 2010. Retrieved from http://apps.who.int/iris/bitstream/10665/43974/1/9789241547598_eng.pdf

23. Kroenke K, Spitzer RL, Williams JBW. The PHQ-9: Validity of a brief depression severity measure. J Gen Intern Med. 2001;16(9):606-13.

24. Spitzer RL, Kroenke K, Williams JBW, Löwe B. A Brief Measure for Assessing Generalized Anxiety Disorder: The GAD-7 Robert L. Arch Intern Med. 2006;166:1092-7.

25. Van Brakel WH, Anderson AM, Mutatkar RK, Bakirtzief Z, Nicholls PG, Raju MS, Das-Pattanayak RK. The Participation Scale: measuring a key concept in public health. Disabil Rehabil. 2006;28(4):193-203.

26. van Brakel W. Participation Scale Users Manual: P-scale Manual. Amsterdam: Participation Scale Development Team; 2010.

27. Attkisson CC, Greenfield TK. The UCSF Client Satisfaction Scales: The Client Satisfaction Questionnaire-8. In: Maruish M, editor. The use of psychological testing for treatment planning and outcome assessment. 3rd ed. Mahwah: Lawrence Erlbaum Associates; 2004

28. Chisholm D, Clark S. Return on investment in global mental health innovation-a primer; prepared for Mental Health Innovation Network; 2016.

29. Kobelt G. Health Economics- an introduction to economic evaluation, 3rd edition, Office of health economics; 2013.

30. Stenberg $\mathrm{K}$, Axelson $\mathrm{H}$, Sheehan $\mathrm{P}$, et al. Advancing social and economic development by investing in women's and children's health: a new Global Investment Framework. Lancet. 2014;383:1333-54.

31. Jamison DT, Summers LH, Alleyne G, et al. Global health 2035: a world converging within a generation. Lancet. 2013:382:1898-955.

32. Atmiyata project films- https://www.youtube.com/channel/UCmMAjMrZ2m_ yhcVL8uh75UA.

33. Chowdhary N, Anand A, Dimidjian S, Shinde S, Weobong B, Balaji M, et al. The Healthy Activity Program lay counsellor delivered treatment for severe depression in India: systematic development and randomized evaluation. $\mathrm{Br}$ J Psychiatry. 2015;208(4):381-8. https://doi.org/10.1192/bjp.bp.114.161075.

34. Chibanda D, Mesu P, Kajawu L, Cowan F, Araya R, Abas MA. Problem-solving therapy for depression and common mental disorders in Zimbabwe: Piloting a task-shifting primary mental health care intervention in a population with a high prevalence of people living with HIV. BMC Public Health. 2011;11(1):828. https://doi.org/10.1186/1471-2458-11-828.
35. Jacobson NS, Dobson KS, Truax PA, et al. A component analysis of cognitive-behavioral treatment for depression. J Consult Clin Psychol. 1996;64:295-304.

36. Martell C, Addis M, Jacobson N. Depression in context: Strategies for quided action. WW Norton \& Co.: New York; 2001

37. Lejuez CW, Hopko DR, Acierno R, Daughters SB, Pagoto SL. Ten Year Revision of the Brief Behavioral Activation Treatment for Depression: Revised treatment manual. Behav Modif. 2011;35(2):111-61.

38. Statistical Software: Release 14. College Station, TX: StataCorp LP.

39. Frost H, Lin Y, Zahs D, Hu M. Cross-cultural survey guidelines: Sample Design. Guidelines for Best Practice in Cross-Cultural Surveys. Ann Arbor: Survey Research Center, Institute for Social Research, University of Michigan; 2016. http://www.ccsg.isr.umich.edu/.

40. Indian Council of Medical Research (ICMR). National Ethical Guidelines for Biomedical and Health Research Involving Human Participants. 2017.

41. Hemming K, Taljaard M, Forbes A. Analysis of cluster randomised stepped wedge trials with repeated cross-sectional samples. Trials. 2017;18(1):101. https://doi.org/10.1186/s13063-017-1833-7.

42. Husereau D, Drummond M, Petrou S, Carswell C, Moher D, Greenberg D, et al. Consolidated Health Economic Evaluation Reporting Standards (CHEERS) - explanation and elaboration: a report of the ISPOR health economic evaluation publication guidelines good reporting practices task force. Value Health. 2013;16:231-50

43. Weijer C, Grimshaw JM, Eccles MP, McRae AD, White A, Brehaut JC, et al. The Ottawa statement on the ethical design and conduct of cluster randomized trials. PLoS Med. 2012;9(11):e1001346. https://doi.org/10.1371/ journal.pmed.1001346

44. The Council for international Organizations of Medical Sciences (CIOMS) in collaboration with the World Health Organization (WHO). International Ethical Guidelines for Health-related Research Involving Humans. Geneva: CIOMS: 2016.

45. Patel V, Araya R, Chowdhary N, et al. Detecting common mental disorders in primary care in India: a comparison of five screening questionnaires. Psychol Med. 2008;38(2):221-8. https://doi.org/10.1017/S0033291707002334.

46. Goldberg DP. The validity of two versions of the GHQ in the WHO study of mental illness in general health care. Psychol Med. 1997;27(1):191-7.

47. Endsley P, Weobong B, Nadkarni A. The psychometric properties of GHQ for detecting common mental disorder among community dwelling men in Goa, India. Asian J Psychiatr. 2017;28:106-10.

\section{Publisher's Note}

Springer Nature remains neutral with regard to jurisdictional claims in published maps and institutional affiliations.

Ready to submit your research? Choose BMC and benefit from:

- fast, convenient online submission

- thorough peer review by experienced researchers in your field

- rapid publication on acceptance

- support for research data, including large and complex data types

- gold Open Access which fosters wider collaboration and increased citations

- maximum visibility for your research: over $100 \mathrm{M}$ website views per year

At $\mathrm{BMC}$, research is always in progress.

Learn more biomedcentral.com/submissions 